\title{
Nuclear stopping for heavy-ion induced reactions in the Fermi energy range: from 1-Body to 2-Body dissipation
}

\author{
O. Lopez ${ }^{1, a}$, G. Lehaut ${ }^{1}$, D. Durand ${ }^{1}$ and M. Aouadi ${ }^{1}$ (INDRA collaboration) \\ ${ }^{1}$ Laboratoire de Physique Corpusculaire de Caen, ENSICAEN, Université de Caen, CNRS-IN2P3, Boule- \\ vard Maréchal Juin, 14050 Caen, France
}

\begin{abstract}
We study the stopping in heavy-ion induced reactions around the Fermi energy in central collisions. The stopping is minimal around the Fermi energy and corresponds to the crossover between the Mean-Field and the nucleonic regimes. This is attributed to the change in the energy dissipation going from 1-body (Mean-Field) to 2-body (nucleonnucleon collisions) dissipation. For this latter, a connection to in-medium transport properties of nuclear matter is proposed and comprehensive values of the nucleon mean free path and nucleon-nucleon cross section are extracted.
\end{abstract}

\section{Introduction}

The study of transport phenomena are of primary importance for understanding the fundamental properties of nuclear matter[1]. They are critical in the description of the supernova collapse and the formation of a neutron star[2]. Transport properties of nuclear matter are also one of the basic ingredients for microscopic models [3, 4]. In this study, we are looking at the global energy dissipation achieved in heavy-ion induced reactions in the Fermi energy domain. We are using the large experimental dataset available in this energy range for symmetric systems recorded with the $4 \pi$ array INDRA[5]. We are looking at central collisions, i.e. collisions corresponding to the maximal overlap and thus leading to the maximal dissipation. Doing so, we will extract information concerning the stopping encountered in such collisions and relate it to the nucleon mean free path and cross section in the nuclear medium.

\section{Stopping for central collisions}

In this work, we are studying INDRA data coming from central collisions, selected through the total multiplicity of charged particle as proposed in [6]. The data span a large body of symmetric systems, with total mass between 70 and 500 mass units, and incident energies covering the full range of the Fermi domain $E_{\text {inc }} / A=10-100 \mathrm{MeV}$. To evaluate the degree of stopping achieved in central collisions, we are using the energy isotropy ratio $R_{E}$ as defined in [6]. This ratio is taking positive values, with 1 for an isotropic emission. We only consider $Z=1$ particles $(p, d, t)$ in this study since we are mainly interested in the properties for nucleon-nucleon collisions. We find here higher $R_{E}$ values than those of [6]. Indeed, the isotropy ratio in [6] was computed by using all charged products,

a e-mail: lopezo@in2p3.fr 
including fragments. It has be shown in [7] that clusterization effects on nucleon phase space lower the isotropy ratio, mainly below $100 \mathrm{~A} \mathrm{MeV}$. We display in Fig. 1 the mean isotropy ratio $<R_{E}>$ calculated for central collisions as a function of incident energy. It is worthwhile to note that in case of asymmetric systems, the incident energy should be conveniently replaced by the total available $\mathrm{cm}$ energy.

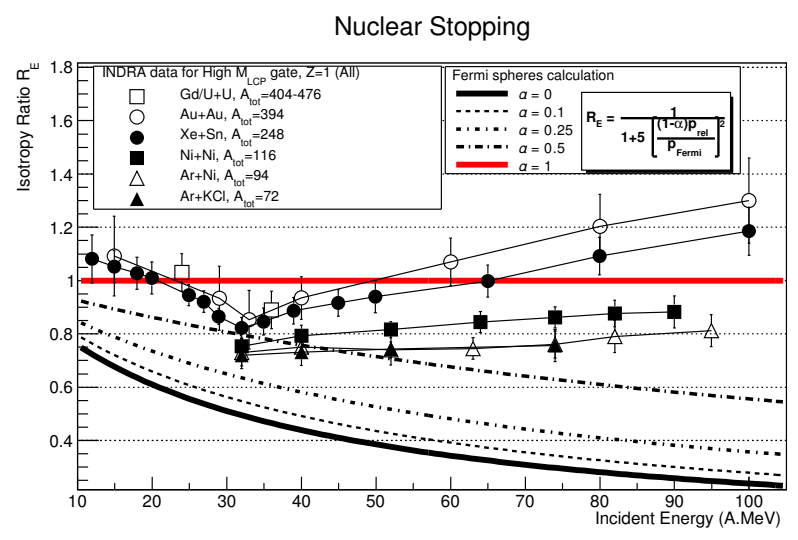

Figure 1. Mean Isotropy ratio $<R_{E}>$ as a function of incident energy for central collisions in symmetric systems recorded with INDRA (symbols). The error bars correspond to statistical + systematic errors.

The coloured curves on Fig. 1 correspond to several analytical calculations associated to the entrance channel; we consider here 2 Fermi spheres separated by the relative momentum $(1-\alpha) p_{\text {rel }}$ corresponding to the relative energy of the entrance channel. $\alpha$ is a free parameter which represents the level of dissipation observed in the collision; $\alpha=1$ (red curve) means full dissipation while $\alpha=0$ (thick black curve) corresponds to the sudden approximation without any dissipation. The displayed error bars correspond to statistical supplemented by an estimation of systematic errors coming from the determination of the mean value [6]. We observe that the closest distance to the no-dissipation scenario $(\alpha=0)$ corresponds to an incident energy slightly below the Fermi energy, around 30 A MeV. Above the Fermi energy, in the nucleonic regime, we can observe a nice mass hierarchy concerning the stopping: the heavier the system, the larger the stopping is. This is fully consistent with a Glauber picture where the degree of stopping is closely related to the number of participants. To get further on this point, we have performed Monte Carlo simulations of 2 Fermi spheres, taking into account nucleon-nucleon collisions through the definition of the nucleon mean free path in the nuclear medium $\lambda_{N N}$. A simple relationship between the degree of stopping and the nucleon mean free path can then be obtained.

\section{Nucleon mean free path in nuclear medium}

By applying this to the experimental data, we obtain the results displayed by Fig. 2. We note that all curves exhibit the same behaviour whatever is the total mass. We observe a maximum at incident energy corresponding to minimal stopping at $E_{\text {inc }} \approx 30 \mathrm{~A} \mathrm{MeV}$ as can be expected from Fig. 1 . The non-physical decrease at low incident energy (below $30 \mathrm{~A} \mathrm{MeV}$ ) can be attributed to the Mean-Field dissipation which is well active in this energy domain, and consequently affects the entrance channel values; in this domain, we should adjust the $\alpha$ parameter to better describe the energy dissipation in the entrance channel. In the limited scope of the present study, we are mainly interested on the nucleonic regime, i.e. incident energy larger than the Fermi energy. Focusing on this incident energy domain, we observe a decrease from $\lambda_{N N} \approx 10 \mathrm{fm}$ to $\lambda_{N N}=5 \mathrm{fm}$ at $E_{\text {inc }} / A \approx 100 \mathrm{MeV}$. It is worthwhile to mention that the value at high incident energy is in agreement with recent microscopic 
Dirac-Brueckner-Hartree-Fock calculations using realistic interactions [8] and hence gives credit to our rather crude determination of the mean free path.

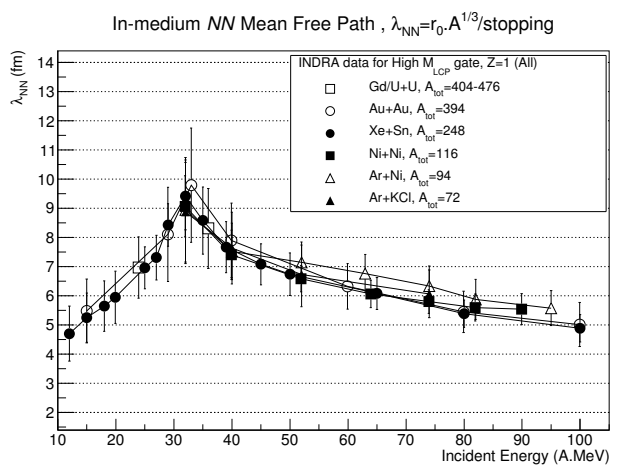

Figure 2. Nucleon mean free path $<\lambda_{N N}>$ as a function of incident energy extracted from experimental data. The errors bars correspond to the statistical+systematic errors.

\section{In-medium effects for nucleon-nucleon cross section}

From the mean free path, we can estimate the nucleon-nucleon cross section by taking the wellknown formula: $\sigma_{N N}=1 /\left(\rho \lambda_{N N}\right)$ where $\rho$ is the nuclear density which varies linearly from $\rho=\rho_{0}$ at $E_{\text {inc }} / A=30 \mathrm{MeV}$ to $\rho=1.5 \rho_{0}$ at $E_{\text {inc }} / A=100 \mathrm{MeV}$ in order to take into account compression effects [1]. To get the real nucleon-nucleon cross section, we have then to correct from the Pauli blocking as a trivial (but essential) 2-body effect in the medium. To do so, we use the prescription of ref. [9]. More sophisticated approaches concerning the Pauli blocking estimation can be found in the litterature [10] but should not change significantly the results. Then, we compute the quenching factor $F=\sigma_{N N}^{i n-m e d i u m} / \sigma_{N N}^{\text {free }}$ by taking the energy-dependent free nucleon-nucleon cross sections $\sigma_{N N}^{\text {free }}$ available in the litterature [11].

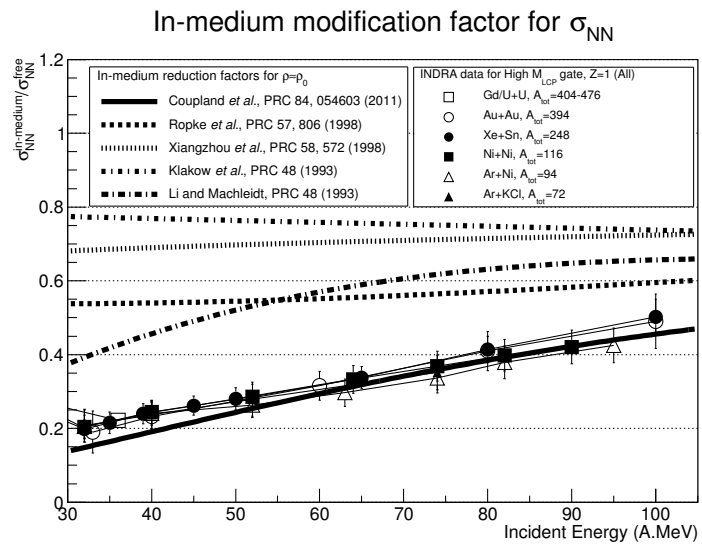

Figure 3. Mean in-medium quenching factor $<F>=\sigma_{N N}^{\text {in-medium }} / \sigma_{N N}^{\text {free }}$ as a function of incident energy for experimental data (symbols) and various theoretical prescriptions (curves). The errors bars for data correspond to the statistical+systematic errors.

The results for the quenching factor $F \mathrm{f}$ are displayed on Fig. 3 for the incident energy range above $E_{\text {inc }} / A=30 \mathrm{MeV}$. The 'experimental' values (symbols) are compared to standard theoretical prescriptions derived from various works and currently used in microscopic transport models [12-16]. We have taken the same linear density dependence as mentioned before to account for compressional effects. We can see that the medium effects, out-of-Pauli blocking, suppress strongly nucleon-nucleon 
collisions at low energy with $F \approx 15 \%$. We obtain values close to $F=50 \%$ at the highest incident energy, here at $100 \mathrm{~A} \mathrm{MeV}$. It is interesting to note that all parametrizations and the data seem to converge toward a common value around $60-70 \%$, indicating that in-medium effects should be quite correctly taken into account above $100 \mathrm{~A} \mathrm{MeV}$ incident energy. For the low energy domain $(30 \mathrm{~A}-100 \mathrm{~A} \mathrm{MeV})$, the parametrizations give quite different results. The best prescription is the one of ref. [16]. It is worthwhile to mention that this parametrization is rather strongly density-dependent and produces large reduction factors compared to other prescriptions [16].

\section{Conclusions}

In this study, we have studied the degree of stopping connected to the energy dissipation in heavyion reactions. We have found a minimal stopping around $E_{\text {inc }}=30 \mathrm{~A} \mathrm{MeV} \mathrm{connected} \mathrm{to} \mathrm{the} \mathrm{crossover}$ between 1-Body to 2-Body dissipation regime. For the latter, we have estimated the nucleon mean free path in the nuclear medium from the degree of stopping achieved in central collisions. The mean free path decreases from $\lambda_{N N} \approx 10 \mathrm{fm}$ at $E_{\text {inc }} / A=30 \mathrm{MeV}$ to $\lambda_{N N}=5 \mathrm{fm}$ at $E_{\text {inc }} / A=100 \mathrm{MeV}$. These values are in agreement with recent theoretical findings using microscopic approaches. The large value relative to the nuclear size $\left(\lambda_{N N}>R\right)$ around the Fermi energy suggests that full thermalization is not achieved in such central collisions [6]. In-medium effects, namely Pauli blocking and high-order correlations, have also been evaluated and are found to be large in the Fermi energy range; it is clear that this energy/density dependence of the nucleon-nucleon cross section has to be properly taken into account in any microscopic transport model used in the Fermi energy range. The best agreement for the in-medium factor $F$ is the one proposed in [16], based on simple geometrical arguments. More detailed analyses of experimental data and comparisons with transport models on stopping properties are indeed welcome to confirm these results.

\section{References}

[1] D. Durand, B. Tamain and E. Suraud, Nuclear Dynamics in the nucleonic regime, Institute Of Physics, New York (2001) and refs. therein

[2] J.M. Lattimer and M. Prakash, The Physics of Neutron Stars, Science 304 (2004) 536

[3] C. Fuchs and H.H. Wolter, Eur. Phys. J. A 30 (2006) 5-21 and refs. therein

[4] A. Andronic et al., Eur. Phys. J. A 30 (2006) 31 and refs. therein

[5] J. Pouthas et al., Nucl. Inst. and Meth. A 357 (1995) 418-442

[6] G. Lehaut et al. (INDRA collaboration), Phys. Rev. Lett. 104 (2010) 232701

[7] G.Q. Zhang et al., Physical Review C 84 (2011) 034612

[8] A. Rios and V. Somà, Phys. Rev. Lett. 108 (2012) 012501

[9] K. Kikuchi and M. Kawai, Nuclear matter and Nuclear Collisions, North Holland, New York (1968)

[10] B. Chen, F. Sammarruca and C.A. Bertulani, [nucl-th] arXiv:1304.6096v1

[11] N. Metropolis et al., Phys. Rev. 110 (1958) 204-220

[12] D. Klakow, G. Welke, and W. Bauer, Phys. Rev. C 48 (1993) 1982-1987

[13] G. Q. Li and R. Machleidt, Phys. Rev. C 48 (1993) 1702

[14] A. Schnell, G. Röpke, U. Lombardo, and H.J.Schulze, Phys. Rev. C 57 (1998) 806-810

[15] C. Xiangzhou et al., Phys. Rev. C 58 (1998) 572-575

[16] D. Coupland et al., Phys. Rev. C 84 (2011) 054603 